\title{
Identification and Expression Pattern of Chemosensory Genes in the Transcriptome of Propsilocerus akamusi
}

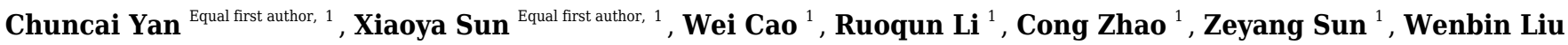 \\ Corresp., 1 , Lina Pan ${ }^{\text {Corresp. } 1}$ \\ ${ }^{1}$ Tianjin Key Laboratory of Animal and Plant Resistance, Tianjin Normal University, Tianjin, China \\ Corresponding Authors: Wenbin Liu, Lina Pan \\ Email address: skylwb@tjnu.edu.cn, skypln@tjnu.edu.cn
}

Chironomidae is the most ecologically diverse insects in aquatic and semi-aquatic habitats. Propsilocerus akamusi (Tokunaga) is a dominant and ubiquitous chironomid species in Eastern Asia and its morphologically unique larvae are also considered as indicator organisms to detect water contamination, potential toxicity and waterborne pathogens. Since few studies to date have focused on the olfactory system of $P$. akamusi, our study aims to elucidate the potential functions of chemosensory genes in P. akamusi . In our study, we found that although signals released from male groups might attract female swarmers, it was a completely male-dominated mating process. Sequencing the transcriptome of $P$. akamusi on an Illumina HiSeq platform generated 4.42, 4.46 and 4.53 Gb of clean reads for heads, legs, and antennae, respectively. 27,609 unigenes, 20,379 coding sequences (CDSs), and 8,073 simple sequence repeats were finally obtained. The gene-level differential expression analysis demonstrated variants among three different tissues, including 2,019 genes specifically expressed in heads, 1,540 genes in legs, and 2,071 genes in antennae. Additionally, we identified an assortment of putative olfactory genes consisting of 34 odorant binding proteins, 17 odorant receptors, 32 gustatory receptors, 22 ionotropic receptors, 6 chemosensory proteins as well as 3 sensory neuron membrane proteins; their relative abundances in the above three tissues were also determined by RT-qPCR. Our finding could allow a more plausible understanding of certain olfaction-mediated behaviors in groups of this macroinvertebrate. 
1 Identification and Expression Pattern of

2 Chemosensory Genes in the Transcriptome of

3 Propsilocerus akamusi

4

5 Chuncai Yan\#, Xiaoya Sun*, Wei Cao, Ruoqun Li, Cong Zhao, Zeyang Sun, Wenbin Liu* and

6 Lina Pan*

7

8 Tianjin Key Laboratory of Animal and Plant Resistance, Tianjin Normal University, Tianjin

$9 \quad 300387$, China

10

Running Title: Transcriptome sequencing of $P$. akamusi

\# Note: Chuncai Yan and Xiaoya Sun contributed equally to this work. 


\section{Abstract}

Chironomidae is the most ecologically diverse insects in aquatic and semi-aquatic habitats.

Propsilocerus akamusi (Tokunaga) is a dominant and ubiquitous chironomid species in Eastern Asia and its morphologically unique larvae are also considered as indicator organisms to detect water contamination, potential toxicity and waterborne pathogens. Since few studies to date have focused on the olfactory system of $P$. akamusi, our study aims to elucidate the potential functions of chemosensory genes in P. akamusi. In our study, we found that although signals released from male groups might attract female swarmers, it was a completely male-dominated mating process. Sequencing the transcriptome of $P$. akamusi on an Illumina HiSeq platform generated 4.42, 4.46 and $4.53 \mathrm{~Gb}$ of clean reads for heads, legs, and antennae, respectively. 27,609 unigenes, 20,379 coding sequences (CDSs), and 8,073 simple sequence repeats were finally obtained. The genelevel differential expression analysis demonstrated variants among three different tissues, including 2,019 genes specifically expressed in heads, 1,540 genes in legs, and 2,071 genes in antennae. Additionally, we identified an assortment of putative olfactory genes consisting of 34 odorant binding proteins, 17 odorant receptors, 32 gustatory receptors, 22 ionotropic receptors, 6 chemosensory proteins as well as 3 sensory neuron membrane proteins; their relative abundances in the above three tissues were also determined by RT-qPCR. Our finding could allow a more plausible understanding of certain olfaction-mediated behaviors in groups of this macroinvertebrate.

\section{Introduction}

Propsilocerus akamusi (Tokunaga) is one of the most ubiquitous chironomid species emerging from numerous eutrophic lakes in Eastern Asia. Acting as prey species for fish and aquatic birds as well as decomposers of plants, this kind of midge is able to connect aquatic and terrestrial food webs (Zheng et al. 2017). Considering the relatively high density and richness of P. akamusi in benthic regions, the presence, absence or quantity of their larvae could be a 
54

55

56

57

58

59

60

61

62

63

valuable indicator for water quality issues at the organism, population, community, and ecosystem levels (Hirabayashi et al. 2003). The term, Chironomidae, is derived from a Greek word for "pantomimist" due to a typical posture that adult insects tend to have their first pair of legs held forward and upward during the rest time (Meigen 1803). The up- and outstretched forelegs are thought to assemble antennae and probably act functionally as sensory organs (Armitage 1995). Males will normally aggregate themselves into a great swarm tending to form above tree-tops, objects and even persons for attracting female ones, which is an extremely common swarm-based mating system in nature (Sæther 1996). Plentiful investigations of $P$. akamusi have been pronounced but most of them primarily focused on its ecology, behavior, karyotype structure, and toxicological response to cadmium stress (Cao et al. 2014; Hirabayashi et al. 2003; Kiknadze et al. 2009; Zheng et al. 2017). However, limited researches to date have been documented regarding the chemosensory system which actually has a critical role in recognizing chemical cues in the surrounding environment. Since the excessive population of $P$. akamusi is problematic to other residents living in the shared community, we assume that a better understanding of the chemosensory system could also enable investigators to seek feasible strategies for pest management. The physiological response to certain chemical stimuli is defined as chemoreception and the roles of which in insects are involved in the process of searching food, locating hospitable ovipostion sites, and socializing with other members. A plethora of chemosensory proteins are categorized into receptors and non-receptor proteins, which are believed to account for chemical communication as well as perception (Leal 2013; Zhao et al. 2016).

Three major groups of the receptor family have been reported, namely odorant receptors (ORs), gustatory receptors (GRs), and ionotropic receptors (IRs). ORs, classified as 7-pass transmembrane proteins, are capable of discriminating distinct odors through its odorant binding sites primarily in sensory neurons. The perception of these chemical stimuli and the consequent intracellular response are associated with multiple behaviors, such as mating, identifying food resources, or alarming conspecies (Venthur \& Zhou 2018). Interface between the surrounding 
81 environment and insect taste system could be successfully established by GRs in gustatory neurons since this $\mathrm{G}$ protein-coupled receptor is able to detect non-volatile information (Venthur \& Zhou 2018). Chemosensory IRs, a novel family evolved from iGluR-related proteins, are thought to accumulate in sensory dendrites and have unique odor-evoked patterns, which means some of their potential ligands are quite different from those for ORs (Rytz et al. 2013).

In addition to the above-mentioned receptor families, odorant binding proteins (OBPs), chemosensory proteins (CSPs), and sensory neuron membrane proteins (SNMPs) (Jia et al. 2018; Wang et al. 2017a) are also involved in the peripheral olfactory recognition and referred to as non-receptor proteins. OBP family contains a large group of divergent members which are soluble and highly abundant in the sensillum hemolymph, a hydrophilic environment where these non-receptor proteins function as necessary transporters for volatile chemicals and deliver them to activate ORs (Leal 2013). However, some OBP genes were proved to have significantly higher expression level in the antennae and the rest vary across different tissues (Liu et al. 2020; Zhang et al. 2020). The CSPs own their names due to the fact that these soluble polypeptides are widely expressed in chemosensory tissues of insects, including the antennae, maxillary, labial palps, and proboscis. According the previous reports, some of them have similar functions as OBPs in initiating biochemical recognition while others could perform tasks unrelated to chemosensation, such as development and transition (Liu et al. 2014; Qu et al. 2020; Waris et al. 2020; Zheng et al. 2020).

Next generation sequencing technology is now routinely applied for identifying candidate genes since it offers a more efficient and cost-effective way than conventional homology-cloning methods. Therefore, this powerful platform has enabled genes involved in chemoreception to be discovered in some insect species without the availability of entire genomes, including Trichogramma chilonis (Liu et al. 2018), Adelphocoris suturalis (Cui et al. 2017), Bemisia tabaci (Wang et al. 2017b), Periplaneta Americana (Chen et al. 2016), Aphis gossypii (Dickens et al. 2014), Spodoptera littoralis (Gu et al. 2015), and Dendroctonus ponderosae (Andersson et al. 2013). 
108

109

110

111

112

113

114

115

116

117

118

119

120

121

122

123

124

125

126

127

128

129

130

131

132

133

With reference to previous researches, we hypothesize that $P$. akamusi behaviors could be more or less manipulated by chemosensory system. Therefore, the genomic information concerning chemoreception and its proper interpretation could offer us valuable insights on this species, which has still been an uncharted territory. Transcriptomic analysis coupled with quantitative real-time PCR were utilized in our study to screen for chemosensory-related genes and their expression patterns, aiming to elucidate the potential functions of these candidate genes.

\section{Materials \& Methods}

\subsection{Insect and tissue collection}

Males of $P$. akamusi midges were collected from Tianjin, China, between October 2017 and November 2017 and then transported live to the lab. All samples were transferred within a few hours of eclosion. The antennae, heads (excluding antennae), and legs were excised from 1500 male specimens under microscope for transcriptome sequencing. The obtained tissues were then immersed in RNAlater (Ambion, AM7020) and stored at $-20^{\circ} \mathrm{C}$ until processing.

\subsection{Olfactometer bioassay}

The emerging $P$. akamusi males tend to congregate into cloud-like swarms which usually appear in the early evening. They seek to mate when females are around or just pass through, thus starting the life cycle. However, we still face deficient knowledge of the sex differences in mate preferences and wonder whether males will take the first step to attract females or not.

A mate selection behavioral test was therefore conducted using a horizontal Y-tube olfactometer consisting of a central glass tube with the length of $115 \mathrm{~mm}$ as well as two lateral glass arms with the length of $75 \mathrm{~mm}$. The diameter for both the tube and arms was $22 \mathrm{~mm}$. The test was performed in a dark room with a small incandescent lamp working as the only light source over the olfactometer. One lateral arm of the olfactometer was selected as the 
134

135

136

137

138

139

140

141

142

143

144

145

146

147

148

149

150

151

152

153

154

155

156

157

158

159

160

experimental arm and the other one is the control. Either the female subjects or the male ones were firstly placed on a piece of filter paper $(10 \times 10 \mathrm{~mm})$ and this paper was then carefully inserted into the midpoint of the experimental arm. Another piece of paper without any subjects was inserted into the equivalent point in the control arm. After the olfactometer was connected to an assembled shunting device, the air sampler was switched on to pump airflow for 30s and then a test individual with the opposite sex was placed at the entrance of the central glass tube. If this test one might prefer to enter into the experimental arm and stayed there for at least $1 \mathrm{~min}$, the chosen arm could be considered as the one with proper pheromones for the insects. However, the midge was allowed to acclimate and make a preference within 5 min. Otherwise, we assumed that no response was given by the insect to the ones with the opposite sex and the trial was ended then. Besides, to prevent light from interfering with the taxis of P. akamusi, the experimental and control arms were swapped after completing the behavioral assays of three individuals. Fifty insects were tested for one trial with triplicates.

\section{3. $R N A$ preparation and $c D N A$ library construction}

The antennae, heads, and legs of 100 male insects were subjected to RNA preparation using TRIzol reagent (Invitrogen, Carlsbad, CA, USA) according to the manufacturer's protocol. After the treatment of DNase I, the mRNA molecules were harvested with Oligo (dT) from the total RNA. The long material of mRNA was then shortened into something compatible for further sequencing with the supplementation of Thermomixer buffer. With TransScript First-Strand cDNA Synthesis SuperMix (TransGen, Beijing, China), the fragmented mRNA next worked as the template for single-strand cDNA which was subsequently synthesized to double strand cDNA. Purification, end reparation, adaptor-ligation and size selection were carried out for final PCR amplification. During the QC (quality control) steps, Agilent 2100 Bioanaylzer and ABI StepOnePlus Real-Time PCR System were used for quantification and qualification of the sample library. The library ended up being sequenced using Illumina HiSeq 4000. 
161

162

163

164

165

166

167

168

169

170

171

172

173

174

175

176

177

178

179

180

181

182

183

184

185

186

\subsection{De novo transcriptome assembly}

After sequencing, a preprocessing of the raw data was performed and consisted of a series of steps, including the trimming of adapter sequences, the removal of reads containing more than $5 \%$ ambiguous bases and the elimination of low quality basis. Here the low quality sequences referred to a situation where a single read comprised over $20 \%$ of bases with Q score less than 15 . The remaining reads generated by these filtering procedures were defined as high quality clean reads and were consequently used for de novo assembly by Trinity v3.0 program (Eisen et al. 1998). This transcript assembly was then clustered into unigenes with the assistance of Tgicl (version: v2.0.6) (de Hoon et al. 2004).

\subsection{Functional annotation of unigenes as well as prediction of coding sequences and simple} sequence repeats

A homology search of functionally annotated unigenes was performed by BLAST (version: v2.2.23, website: http://blast.ncbi.nlm.nih.gov/Blast.cgi) against NCBI non-redundant protein sequences (NR), non-redundant nucleotide sequences (NT), Clusters of Orthologous Groups of Proteins (COG), Kyoto Encyclopedia of Genes and Genomes (KEGG), Gene Ontology (GO) and Swiss-Prot databases (Altschul et al. 1990; Conesa et al. 2005). Besides, InterPro analysis was measured by InterProScan5 (version: v5.11-51.0, website: https://code.google.com/p/interproscan/wiki/Introduction) for providing predictive information of gene families and their critical domains (Quevillon et al. 2005). TransDecoder (https:/github.com/TransDecoder/TransDecoder/releases) was utilized to recognize candidate coding regions and peptide sequences within the Trinity-created transcript assembly. Unigenes that could not be aligned to any of the databases mentioned above were later predicted by ESTScan (Saldanha 2004).

\subsection{Differentially expressed gene analysis}

Peer) reviewing PDF | (2020:03:46684:1:1:NEW 18 Jun 2020) 
187

188

189

190

191

192

193

194

195

196

197

198

199

200

201

202

203

204

205

206

207

208

209

210

211

212

The clean reads were mapped to unigenes using Bowtie2 (Langmead \& Salzberg 2012), and the expression levels of these high-quality unigene sets were computed based on RSEM values (Li \& Dewey 2011). Poisson distribution was applied to accurately describe gene expression variation on those with more than 2 -fold changes as well as less than 0.001 false discovery rate [FDR] (Audic \& Claverie 1997). The performance of hierarchical clustering enabled the differentially expressed genes (DEGs) with similar features to be partitioned into distinct clusters and this analysis was done by having the aid of pheatmap function in R. When two or more relatively homogeneous groups were clustered, the intersection and union DEGs between them were performed. The DEGs were classified according to the GO and KEGG annotation results; FDR was calculated for each p-value. In general, the terms with FDR no more than 0.001 were defined as significantly enriched ones.

\subsection{Identification of chemosensory genes}

To identify candidate chemosensory unigenes for $P$. akamusi, an analysis using the tBLASTn modules was performed with reference to all the publicly available sequences of OBP, OR, CSP, GR, IR, and SNMP from Diptera species. Meanwhile, all the candidates were manually checked by using the BLASTx program.

\section{8. $R T-q P C R$ analysis}

Using previously obtained cDNA from the three body parts of $P$. akamusi (the antennae, heads excluding antennae, and legs) as templates, OBPs, ORs, CSPs, GRs, IRs, and SNMPs were selected for RT-qPCR analysis. Specific primer pairs were designed by Primer5 according to the transcriptome data (Table S1). The RT-qPCR was conducted on the Roche LightCycler 480 detector (Stratagene, La Jolla, CA, USA) with the following cycling parameters: $94^{\circ} \mathrm{C}$ for 30 $\mathrm{s}, 40$ cycles of $94^{\circ} \mathrm{C}$ for $5 \mathrm{~s}, 55^{\circ} \mathrm{C}$ for $10 \mathrm{~s}$, and $72^{\circ} \mathrm{C}$ for $10 \mathrm{~s}$. The dissociation curves were carried out as a post-qPCR analysis, during which all the components were firstly denatured for 
$2135 \mathrm{~s}$ at $95^{\circ} \mathrm{C}$, followed by cooling to $60^{\circ} \mathrm{C}$ for $1 \mathrm{~min}$. An increase to $95^{\circ} \mathrm{C}$ for $30 \mathrm{~s}$ then took place,

214 followed by cooling to $50{ }^{\circ} \mathrm{C}$ for $30 \mathrm{~s}$. The relative gene expression data were analyzed by

215 normalizing the threshold cycle $(\mathrm{Ct})$ value of each sample to that of endogenous $\beta$-tubulin, which

216 was determined with $2^{-\Delta \Delta \mathrm{Ct}}$ method (Livak \& Schmittgen 2001). The gene amplification from

217 each tissue part of $P$. akamusi was actually completed in triplicates and the statistical

218 significance was examined with one-way ANOVA test.

219

220

2.9 Phylogenetic analysis and classification of Chemosensory Genes

221

222

223

224

225

226

227

228

229

230

231

232

233

234

235

236

237

238

A set of chemosensory gene sequences from different Dipteran species were retrieved from the GenBank database, including Drosophila melanogaster, Aedes aegypti, Anopheles gambiae and Culex quinquefasciatus. To visually demonstrate the relationship between candidate chemosensory genes from $P$. akamusi and other dipteran species, they were first joined with alignments by Muscle 3.8.31 (Edgar 2004) with default option and then manually refined by BioEdit v7.2.5 (Hall 1999). The unrooted phylogenetic trees of each family were then generated through running MEGA 7.0 (Kumar et al. 2016) and using neighbor joining (NJ) method with poisson correction, pairwise deletion, and rapid bootstraps (1000 replicates).

\section{Results}

\subsection{Mate selection behavioral test}

To examine whether the male individuals will attempt to mate with female ones, a female insect was placed in the experimental corridor of the Y-tube olfactometer while a male was placed at the open end of the central glass tube. The result showed that the majority of males were relatively active with approximately 58\% choosing the female-occupied corridor and 38\% choosing the empty side (Fig. 1A). However, this obvious response was not observed when females were given the choice to make a behavioral preference. It turned out that $82 \%$ female insects were recorded as non-responders to either corridor and the probability of females 
239

240

241

242

243

244

245

246

247

248

249

250

251

252

253

254

255

256

257

258

259

260

261

262

263

264

selectively orientating to the arm with male subjects was only $8 \%$ (Fig. 1B). These results revealed that males tended to seek companions and dominated the mating process probably because of the exposure to airborne pheromones released from the females. We therefore selected male individuals as the main subject for our further investigations and tried to explore how exactly the mutual attraction between the sexes occur during mating.

\subsection{Transcriptome sequencing and de novo assembly}

With the support of high-throughput sequencing platform, a set of raw sequencing reads were constructed from three different tissues of $P$. akamusi males, including $50.20 \mathrm{Mb}$ from heads (excluding antennae), 51.82 $\mathrm{Mb}$ from antennae and 55.06 $\mathrm{Mb}$ from legs (Table 1). The trimming and elimination of adaptor-polluted and low-quality basis led to the generation of $4.42 \mathrm{~Gb}, 4.46 \mathrm{~Gb}$, and $4.53 \mathrm{~Gb}$ clean reads from heads, legs, and antennae, respectively. The data also showed that the assessed intrinsic quality of the clean reads was sufficient for further analysis (Table 2) and the transcript length distribution was identified as well (Fig. S1, S2). In general, 27,609 unigenes were assembled with a total length, mean length, N50, and GC content of $28,312,956,1,025 \mathrm{bp}, 1,832 \mathrm{bp}$ and $38.23 \%$, respectively (Table 3 ).

\subsection{Functional annotation and classification of the unigenes}

The unigenes were annotated in accordance to seven functional databases and the result of this comparison illustrated that 17,311 unigenes $(62.70 \%)$ were markedly matched with published proteins in NR database. Similarly, the total of annotated unigenes against known information from NT, Swiss-Prot, COG, KEGG, GO and InterPro was 7,936 (28.74\%), 14,614 (52.93\%), 7,986 (28.93\%), 14,341 (51.94\%), 7,501 (27.17\%) and 14,752(53.43\%), respectively (Table 4).

The homology searching against NR database revealed that the annotated sequences of $P$. akamusi were partly matched to sequences of Aedes aegypti (2,769 matching hits, 16.00\%), 
265

266

267

268

269

270

271

272

273

274

275

276

277

278

279

280

281

282

283

284

285

286

287

288

289

290

291

followed by Aedes albopictus (1,870 matching hits, 10.80\%), Culex quinquefasciatus (1,741 matching hits, 10.06\%) and Anopheles gambiae str. (1,311 matching hits, 7.57\%) (Fig. 2).

GO analysis, an enrichment tool, rendered 42,572 unigenes to be grouped into three functional categories, i.e. Biological Process (18,955 unigenes), Cellular Component (15,232 unigenes), and Molecular Function (8,385 unigenes). There were 25 subcategories in Biological Process with 'cellular process' accounting for $22.2 \%$ (4,213 unigenes) followed by 'metabolic process' making up 18\% (3,411 unigenes). 19 sub-categories were included in the classification of Cellular Component and among which 'groups of cell' (3,145 unigenes, 20.6\%) and 'cell parts' $(3,121$ unigenes, 20.5\%) were the most abundant GO terms. For Molecular Function, sequences were predominately assigned to 'catalytic activity' (3,309 unigenes, 39.5\%) and 'binding' (3, 267 unigenes, 39\%) (Fig. 3A).

A more detailed comprehension of gene biochemical function could be further gained via KEGG analysis. The result delineated that a total of 25,377 unigenes were mapped and organized to six functional clusters, including Cellular Process (2,476 unigenes), Environmental Information Processing (2,613 unigenes), Genetic Information Processing (2,531 unigenes), Human Disease (6,293 unigenes), Metabolism (6,405 unigenes), and Organismal System (5,059 unigenes) (Fig. 3B).

Besides, potential functions of these putative unigenes were predicted with the assistance of COG database which was considered as a useful tool for understanding the orthologous relationships of gene products. The retrieved unigene sets were classified under 25 categories, among which the cluster of 'General function prediction only' (2,338 unigenes, 19\%), 'Translation, ribosomal structure and biogenesis' (979 unigenes, 8\%) and 'Posttranslational modification, protein turnover, chaperones' (941 unigenes, $7.7 \%$ ) were the most representative three classifications (Fig. 3C). Venn diagram aimed at describing the similarities and differences of unigenes when searched against NR, COG, KEGG, Swissprot and Interpro databases and it turned out that 6,766 unigenes overlapped as the intersection proportion (Fig. 3D). 
292

293

294

295

296

297

298

299

300

301

302

303

304

305

306

307

308

309

310

311

312

313

314

315

316

\subsection{CDS prediction}

17,522 CDSs were screened out from the 27,609 annotated unigenes. Since ESTScan program was capable of constructing CDSs in the remaining unigenes which were not of particular matches with the aforementioned databases, a total of 2,857 CDSs were predicted from these unannotated unigenes. Altogether, 20,379 CDSs were predicted from unigenes with a total length, a mean length, N50 and GC content of 16,667,634 bp, 817 bp, 1,290 bp and $42 \%$, respectively (Fig. 4, Table 5).

\subsection{Differentially expressed genes}

Differentially expressed genes were examined by comparison among heads, legs, and antennae of male P. akamusi midges (Fig. 5A). The gene expression profiling analysis demonstrated that there were 2,281 up-regulated along with 4,865 down-regulated genes in heads against legs. A total of 3,421 were expressed at a markedly higher level while 5,792 genes were at lower levels in antennae vs. heads. Plus, 2,066 genes were expressed with greater numbers while 7,093 genes with relatively smaller numbers in antennae vs. legs. Shared and exclusively expressed genes among three tissues were shown in Fig. 5B. The number of genes differentially expressed among three tissue types was quite similar to each other with 21,103 in heads, 20,980 in legs, and 21,750 in antennae. Meanwhile, a subset of 5,620 genes that differentially expressed were identified with a tissue-dependent manner, including 2019 specifically expressed in head, 1540 genes expressed in legs and 2071 expressed in antennae.

\subsection{Candidate odorant binding proteins}

Based on functional annotation and tBLASTn results with an E-value of 1E-5 or lower (Table S2), 34 transcripts ranging from 138 to $927 \mathrm{bp}$ were isolated as best candidate OBPs (PakaOBPs) in the P. akamusi transcriptome, and 29 of which contained full-length open reading 
317 frames (ORFs). The identified PakaOBP transcripts together with corresponding sequence data 318 of OBPs from A. gambiae, C. quinquefasciatus, D. melanogaster and A. aegypti were used for 319 obtaining phylogenetic inferences, as depicted in Fig. S3.

320 To determine the transcriptional output of these candidate genes among different tissues, 12 321 PakaOBPs with RPKM>1.2 were selected for RT-qPCR amplification and the expression profile 322 of each gene differed. Five OBPs (PakaOBP1, 5, 8, 9 and 10) had noticeably higher proportion in 323 antennae than either heads or legs. Meanwhile, seven other OBPs (PakaOBP1, 2, 3, 4, 6, 7, and 12) were expressed with a markedly greater numbers in legs. Of note, we found a unique antennae-specific expression pattern of PakaOBP9 since this particular gene showed almost no evidence of presence in other tissues (Fig. 6).

\subsection{Candidate odorant receptors}

Putative OR genes of $P$. akamusi (PakaORs) were represented based on their similarities to known insect ORs and tBLASTn results with an E-value of 1E-5 or lower generated a total of 17 PakaORs with length ranging from 312 to 1671 bp (Table S3). Among them, 16 sequences were available as full-length coding ones. Evolutionary distances were evaluated among ORs from the sampled P. akamusi and four other Dipteran species, as shown in Fig. S4.

\subsection{Candidate gustatory receptors}

A total of 32 candidate GR genes (PakaGRs) were identified based on the tBLASTn results 
343 phylogenetic tree was constructed by using concatenated orthologous sequences derived from 344 GRs in different Dipteran species (Fig. S5).

345 The relatively abundant transcripts (PakaGR1-10) according to the RPKM analysis were

subjected to RT-qPCR detection, aiming to figure out the particular tissues where they preferred to be expressed. It turned out that PakaGR1 and PakaGR2 were mainly expressed in the antennae whereas PakaGR3 had a significant quantity in legs. Meanwhile, a pervasive expression pattern was shown for 10 other PakaGRs, including PakaGR2 and PakaGR3-10 (Fig. 8).

\subsection{Candidates for other chemosensory genes}

Our bioinformatic data facilitated the discovery of 22 transcripts for candidate IR genes (PakaIRs), 6 for candidate CSP genes (PakaCSPs), and 3 for candidate SNMP genes (PakaSNMPs) with the result of tBLASTn being E-value of 1E-5 or lower; of these genes, 19 PakaIRs, 2 PakaCSPs, and 3 PakaSNMPs included full length ORFs (Table S5 and S6). The phylogenetic signals of PakaIRs, PakaCSPs, and PakaSNMPs from P. akamusi with those from other four species were mapped onto the tree, as described in Fig. S8.

RT-qPCR data revealed that 16 PakaIRs were highly expressed in heads and legs (Fig. 9) while CSP1 was distributively expressed at varying levels in all tissues. Additionally, there was an abundance of PakaSNMP1 presented in antennae and legs at transcript level, which was a reliable clue for understanding its role in chemosensory process.

\section{Discussion}

The Chironomidae chemoreception is barely explored at either genetic or molecular levels, especially in comparison to some other Dipterans, such as Culicidae and Drosophila, which are commonly selected as subjects in researches of chemosensation.

Next generation sequencing technology was utilized in our study to yield a diverse array of candidate chemoreception-related genes of $P$. akamusi, a dominant Chironomidae species in 
369 freshwater environment. The sequencing data presented six categories of putative chemosensory 370 genes, including 34 PakaOBPs, 17 PakaORs, 32 PakaGRs, 22 PakaIRs, 6 PakaCSPs, and 3

371 PakaSNMPs (Fig. 6-10), which was the first known attempt for the identification of 372 chemosensory genes in P. akamusi. However, it turned out that the number of these identified 373 genes was quite tiny when compared to that of genes from other species. For instance, 82 ORs, 374 77OBPs, 30GRs, and 102IRs have previously been collected from Aedes albopictus. Strictly 375 speaking, we have to admit that most of our transcripts did not comprise complete ORFs.

376 The genome sequencing in our work facilitated an identification of 34 PakaOBPs, which 377 was fewer than the numbers reported from other species, including Bombyxmori (44) (Gong et al. 378 2009), A. gambiae (57) (Biessmann et al. 2002; Vogt 2002; Xu et al. 2003), D. melanogaster (51) 379 (Hekmat-Scafe 2002), and Agrotis ipsilon (33) (Gu et al. 2013). We assume that the less quantitative sufficiency of OBPs in various tissues of $P$. akamusi accompanied with barely detectable levels could be one possible explanation. Besides, the lack of some host-seeking cues in lab could be another factor for this limited number since OBPs will be normally stimulated with the exposure to proper odors in the nature. The tissue-specific patterns of candidate OBPs could provide essential clues for gene function. For example, we observed that PakaOBP9 was specifically expressed in the antennae whereas most PakaOBPs repertoire did not apparently display an antenna-biased expression profile (Fig. 6), suggesting a likely association with certain chemosensory reactions. Besides, PakaOBP1 was abundantly expressed in antennae whereas PakaOBP5 was highly expressed in heads and antennae (Fig. 6). One previous study has reported that OBP21 was highly expressed in a non-olfactory system, like the venom gland of Apis mellifera, and hypothesized that this molecule might serve as a carrier of potential ligands other than odorants ( $\mathrm{Li}$ et al. 2013; Pelosi et al. 2018). However, the expression profile of PakaOBPs in a non-olfactory part still remains vague and requires a further exploration to better appreciate the function of this gene.

The ORs, belonging to the superfamily of G-protein coupled receptors, are critical 395 recognition elements involved in olfactory sensory system. We have analyzed the whole genome 
396 397

398

399

400

401

402

403

404

405

406

407

408

409

410

411

412

413

414

415

416

417

418

419

420

421

422

to prospect candidate ORs and finally recognized as many as 11 transcripts in P. akamusi, which is actually far fewer than 170 genes found in Apis mellifera (Robertson \& Wanner 2006) and 60 in the parasitoids Microplitis mediator (Wang et al. 2015) but greater than 6 discovered in Cotesia vestalis (Matsunami et al. 2012b). Most of newly PakaORs transcripts showed a significant enrichment in the antennae whereas PakaOR3-6 were predominantly detected in tissues like heads. The expression pattern of ORs in non-olfactory tissues suggests they might have some undefined physiological functions.

GRs, regarded as critical chemoreceptors, usually endow gustatory receptor neurons with the capability to perceive soluble tastes and respond to carbon dioxide. This kind of peripheral receptor comprises more conserved sequences and structures among diverse species when compared to ORs. The de novo assembly in our work allowed a total of 32 PakaGRs to be captured, the same number as identified in A. Albopictus. Despite the typical antenna-specific expression pattern of GRs in most insect species, our discovery revealed that most PakaGRs were significantly enriched in legs (PakaGR2, 3, 5, 6, 7, 9, and 10) or heads (PakaGR5 and 9). Since $P$. akamusi posturing with forelegs is a common phenomenon in nature, we wonder whether the high abundance of ORs in legs might have potential roles in chemosensory-related perception.

IRs are novel families of highly divergent ionotropic glutamate receptors and broader attentions with respect to this group have been constantly received. 66 have been reported in $D$. melanogaster (Benton et al. 2009), 21 in Manduca sexta (Koenig et al. 2015), 15 in Cydia pomonella (Matsunami et al. 2012a), and 12 in Helicoverpa armigera (Dickens et al. 2015). Although IRs have been reported to exist in various insect genomes or transcriptomes, their function has only been hinted in studies focusing on Drosophila. There is a considerable discrepancy between the environmental stimuli recognized by ORs and IRs. In a combinatorial fashion, IRs could provide a strong response to a broad range of odors which, however, only induce weak or even no answer of ORs. We have identified a set of 22 IRs in the genome of $P$. akamusi compared to transcriptomes of D. melanogaster with 66 IRs (Benton et al. 2009), 
423 Manduca sexta with 21 IRs (Koenig et al. 2015), Cydia pomonella with 15 IRs (Matsunami et al. 424 2012), and Helicoverpa armigera with 12 IRs (Dickens et al. 2015). A comprehensive map of 425 their expressions was generated with reference to RPKM values and we found that PakaIR3, 4, 7, $4268,9,10,12$, and 13 showed high transcript levels in heads and legs while none in antennae. Plus, 427 the expression levels of these PakaIRs are higher than those of PakaOBPs and PakaCSPs, 428 indicating possible protein functions in chemosensory processes in these non-olfactory parts.

\section{Conclusions}

In our study, the transcriptome of $P$. akamusi was sequenced by using Illumina Hiseq platform by which 27,609 unigenes, 20,379 CDSs, and 8,073 simple sequence repeats were obtained. The differentially expressed gene analysis showed that there were 2,019 head-specific genes, 1,540 leg-specific genes, and 2,071 antennae-specific genes. Furthermore, candidate olfactory-related genes were identified and their relative abundances in the above tissues were examined by RT-qPCR as well. In general, 34 odorant binding proteins, 17 odorant receptors, 32 gustatory receptors, 22 ionotropic receptors, 6 chemosensory proteins as well as 3 sensory neuron membrane proteins were collected.

\section{Acknowledgements}

This project was supported by the National Natural Science Foundation of China (No. 31702058, 442 31672324, 31801994); Natural Science Foundation of Tianjin (No. 18JCYBJC96300, 443 17JCQNJC14900, 14JCQNJC14600, 18JCQNJC14700) and Tianjin Normal University 444 Foundation (5RL104, 043135202-XB1715，52XB1003，52XB1005， 043135202-XK1706, 445 135305JF79).

446

447 Data availability

448 All unigenes of $P$. akamusi used in this study were available in the supplementary file (All- 
449

450

451

452

453

454

455

456

457

458

459

460

461

462

463

464

465

466

467

468

469

470

471

472

473

474

475

476

477

478

479

480

481

482

483

484

485

486

Unigene. fa) and GenBank database. The list and accession numbers of candidate olfactoryrelated genes of $P$. akamusi were listed in the supplementary Table (Table S2-S6).

\section{References}

Altschul SF, Gish W, Miller W, Myers EW, and Lipman DJ. 1990. Basic local alignment search tool. Journal of Molecular Biology 215:403-410. 10.1016/s0022-2836(05)80360-2

Andersson MN, Grosse-Wilde E, Keeling CI, Bengtsson JM, Yuen MMS, Li M, Hillbur Y, Bohlmann J, Hansson BS, and Schlyter F. 2013. Antennal transcriptome analysis of the chemosensory gene families in the tree killing bark beetles, Ips typographus and Dendroctonus ponderosae (Coleoptera: Curculionidae: Scolytinae). BMC Genomics 14:198. 10.1186/1471-2164-14-198

Armitage PD. 1995. Behaviour and ecology of adults. In: Armitage PD, Cranston, P. S., Pinder, L. C. V., editor. The Chironomidae Biology and ecology of non-biting midges. London: Chapman and Hall. p 194-224.

Audic S, and Claverie J-M. 1997. The Significance of Digital Gene Expression Profiles. Genome Research 7:986-995. 10.1101/gr.7.10.986

Benton R, Vannice KS, Gomez-Diaz C, and Vosshall LB. 2009. Variant Ionotropic Glutamate Receptors as Chemosensory Receptors in Drosophila. Cell 136:149-162. 10.1016/j.cell.2008.12.001

Biessmann H, Walter MF, Dimitratos S, and Woods D. 2002. Isolation of cDNA clones encoding putative odourant binding proteins from the antennae of the malariatransmitting mosquito, Anopheles gambiae. Insect Molecular Biology 11:123-132. 10.1046/j.1365-2583.2002.00316.x

Cao CW, Niu F, Li XP, Ge SL, and Wang ZY. 2014. Acute and joint toxicity of twelve substituted benzene compounds to Propsilocerus akamusi Tokunaga. Central European Journal of Biology 9:550-558.

Chen Y, He M, Li Z-Q, Zhang Y-N, and He P. 2016. Identification and tissue expression profile of genes from three chemoreceptor families in an urban pest, Periplaneta americana. Scientific Reports 6. 10.1038/srep27495

Conesa A, Gotz S, Garcia-Gomez JM, Terol J, Talon M, and Robles M. 2005. Blast2GO: a universal tool for annotation, visualization and analysis in functional genomics research. Bioinformatics 21:3674-3676. 10.1093/bioinformatics/bti610

Cui H-H, Gu S-H, Zhu X-Q, Wei Y, Liu H-W, Khalid HD, Guo Y-Y, and Zhang Y-J. 2017. Odorant-binding and chemosensory proteins identified in the antennal transcriptome of Adelphocoris suturalis Jakovlev. Comparative Biochemistry and Physiology Part D: Genomics and Proteomics 24:139-145. 10.1016/j.cbd.2016.03.001

de Hoon MJ, Imoto S, Nolan J, and Miyano S. 2004. Open source clustering software. 
487

488

489

490

491

492

493

494

495

496

497

498

499

500

501

502

503

504

505

506

507

508

509

510

511

512

513

514

515

516

517

518

519

520

521

522

523

524

525

526

527

Bioinformatics 20:1453-1454. 10.1093/bioinformatics/bth078 [pii]

Dickens JC, Cao D, Liu Y, Walker WB, Li J, and Wang G. 2014. Molecular Characterization of the Aphis gossypii Olfactory Receptor Gene Families. Plos One 9:e101187. 10.1371/journal.pone.0101187

Dickens JC, Gu X-C, Zhang Y-N, Kang K, Dong S-L, and Zhang L-W. 2015. Antennal Transcriptome Analysis of Odorant Reception Genes in the Red Turpentine Beetle (RTB), Dendroctonus valens. Plos One 10:e0125159. 10.1371/journal.pone.0125159

Edgar RC. 2004. MUSCLE: a multiple sequence alignment method with reduced time and space complexity. BMC Bioinformatics 5:113. 10.1186/1471-2105-5-113 [pii]

Eisen MB, Spellman PT, Brown PO, and Botstein D. 1998. Cluster analysis and display of genome-wide expression patterns. Proc Natl Acad Sci U S A 95:14863-14868.

Gong D-P, Zhang H-J, Zhao P, Xia Q-Y, and Xiang Z-H. 2009. The Odorant Binding Protein Gene Family from the Genome of Silkworm, Bombyx mori. BMC Genomics 10:332. 10.1186/1471-2164-10-332

Gu S-H, Zhou J-J, Gao S, Wang D-H, Li X-C, Guo Y-Y, and Zhang Y-J. 2015. Identification and comparative expression analysis of odorant binding protein genes in the tobacco cutworm Spodoptera litura. Scientific Reports 5. 10.1038/srep13800

Gu S-H, Zhou J-J, Wang G-R, Zhang Y-J, and Guo Y-Y. 2013. Sex pheromone recognition and immunolocalization of three pheromone binding proteins in the black cutworm moth Agrotis ipsilon. Insect Biochemistry and Molecular Biology 43:237-251. 10.1016/j.ibmb.2012.12.009

Hall T. 1999. BioEdit: A user-friendly biological sequence alignment program for Windows 95/98/NT. Nucleic Acids Symposium Series 41:95-98.

Hekmat-Scafe DS. 2002. Genome-Wide Analysis of the Odorant-Binding Protein Gene Family in Drosophila melanogaster. Genome Research 12:1357-1369. 10.1101/gr.239402

Hirabayashi K, Hanazato T, Ogawara M, Sakuma M, and Nakamoto N. 2003. Long-term investigation of Propsilocerus akamusi (Tokunaga) (Diptera, Chironomidae) from a shallow eutrophic lake, Suwa, in Central Japan : An attempt to forecast the massive emergence of adult midges. Medical Entomology \& Zoology 54.

Jia X, Zhang X, Liu H, Wang R, and Zhang T. 2018. Identification of chemosensory genes from the antennal transcriptome of Indian meal moth Plodia interpunctella. Plos One 13:e0189889. 10.1371/journal.pone.0189889

Kiknadze II, Wang XH, and Istomina AG. 2009. Karyotype of Propsilocerus akamusi (Tokunaga) from China (Diptera : Chironomidae). Zootaxa 765:1-8.

Koenig C, Hirsh A, Bucks S, Klinner C, Vogel H, Shukla A, Mansfield JH, Morton B, Hansson BS, and Grosse-Wilde E. 2015. A reference gene set for chemosensory receptor genes of Manduca sexta. Insect Biochemistry and Molecular Biology 66:51-63. 10.1016/j.ibmb.2015.09.007

Kumar S, Stecher G, and Tamura K. 2016. MEGA7: Molecular Evolutionary Genetics Analysis Version 7.0 for Bigger Datasets. Molecular Biology and Evolution 33:1870-1874. $10.1093 / \mathrm{molbev} / \mathrm{msw} 054$ 
528 Langmead B, and Salzberg SL. 2012. Fast gapped-read alignment with Bowtie 2. Nature

529

530

531

532

533

534

535

536

537

538

539

540

541

542

543

544

545

546

547

548

549

550

551

552

553

554

555

556

557

558

559

560

561

562

563

564

565

566

567

568 Methods 9:357-359. 10.1038/nmeth.1923

Leal WS. 2013. Odorant reception in insects: roles of receptors, binding proteins, and degrading enzymes. Annu Rev Entomol 58:373-391. 10.1146/annurev-ento-120811-153635

Li B, and Dewey CN. 2011. RSEM: accurate transcript quantification from RNA-Seq data with or without a reference genome. BMC Bioinformatics 12:323. 10.1186/1471-2105-12-323

Li R, Zhang L, Fang Y, Han B, Lu X, Zhou T, Feng M, and Li J. 2013. Proteome and phosphoproteome analysis of honeybee (Apis mellifera) venom collected from electrical stimulation and manual extraction of the venom gland. BMC Genomics 14:766. 10.1186/1471-2164-14-766

Liu J-B, Wu H, Yi J-Q, Song Z-W, Li D-S, and Zhang G-R. 2018. Transcriptome characterization and gene expression analysis related to chemoreception in Trichogramma chilonis, an egg parasitoid. Gene. 10.1016/j.gene.2018.07.065

Liu YL, Guo H, Huang LQ, Pelosi P, and Wang CZ. 2014. Unique function of a chemosensory protein in the proboscis of two Helicoverpa species. J Exp Biol 217:1821-1826. 10.1242/jeb.102020 jeb.102020 [pii]

Liu Z, Liang X-F, Xu L, Keesey IW, Lei Z-R, Smagghe G, and Wang J-J. 2020. An AntennaeSpecific Odorant-Binding Protein Is Involved in Bactrocera dorsalis Olfaction. Frontiers in Ecology and Evolution 8. 10.3389/fevo.2020.00063

Livak KJ, and Schmittgen TD. 2001. Analysis of relative gene expression data using real-time quantitative PCR and the 2(-Delta Delta C(T)) Method. Methods 25:402-408. 10.1006/meth.2001.1262 S1046-2023(01)91262-9 [pii]

Matsunami H, Bengtsson JM, Trona F, Montagné N, Anfora G, Ignell R, Witzgall P, and Jacquin-Joly E. 2012a. Putative Chemosensory Receptors of the Codling Moth, Cydia pomonella, Identified by Antennal Transcriptome Analysis. Plos One 7:e31620. 10.1371/journal.pone.0031620

Matsunami H, Nishimura O, Brillada C, Yazawa S, Maffei ME, and Arimura G-i. 2012b. Transcriptome Pyrosequencing of the Parasitoid Wasp Cotesia vestalis: Genes Involved in the Antennal Odorant-Sensory System. Plos One 7:e50664. 10.1371/journal.pone.0050664

Meigen JW. 1803. Versuch einer neuen GattungsEintheilung [sic!] der europäischen zweiflügligen Insekten. Magazin für Insektenkunde Brunswick 2:259-281.

Pelosi P, Iovinella I, Zhu J, Wang G, and Dani FR. 2018. Beyond chemoreception: diverse tasks of soluble olfactory proteins in insects. Biological Reviews 93:184-200. $10.1111 /$ brv.12339

Qu MQ, Cui Y, Zou Y, Wu ZZ, and Lin JT. 2020. Identification and expression analysis of odorant binding proteins and chemosensory proteins from dissected antennae and mouthparts of the rice bug Leptocorisa acuta. Comp Biochem Physiol Part D Genomics Proteomics 33:100631. S1744-117X(19)30160-1 [pii] 10.1016/j.cbd.2019.100631

Quevillon E, Silventoinen V, Pillai S, Harte N, Mulder N, Apweiler R, and Lopez R. 2005. InterProScan: protein domains identifier. Nucleic Acids Research 33:W116-W120. 
569

570

571

572

573

574

575

576

577

578

579

580

581

582

583

584

585

586

587

588

589

590

591

592

593

594

595

596

597

598

599

600

601

602

603

604

605

606

607

608

609

\subsection{3/nar/gki442}

Robertson HM, and Wanner KW. 2006. The chemoreceptor superfamily in the honey bee, Apis mellifera: Expansion of the odorant, but not gustatory, receptor family. Genome Research 16:1395-1403. 10.1101/gr.5057506

Rytz R, Croset V, and Benton R. 2013. Ionotropic Receptors (IRs): Chemosensory ionotropic glutamate receptors in Drosophila and beyond. Insect Biochemistry and Molecular Biology 43:888-897. 10.1016/j.ibmb.2013.02.007

Sæther OA, Wang, X. 1996 Revision of the orthoclad genus Propsilocerus Kieffer (= Tokunagayusurika Sasa) (Diptera: Chironomidae) Ent Scand 27:441-479.

Saldanha AJ. 2004. Java Treeview--extensible visualization of microarray data. Bioinformatics 20:3246-3248. 10.1093/bioinformatics/bth349

Venthur H, and Zhou J-J. 2018. Odorant Receptors and Odorant-Binding Proteins as Insect Pest Control Targets: A Comparative Analysis. Frontiers in Physiology 9. 10.3389/fphys.2018.01163

Vogt RG. 2002. Journal of Chemical Ecology 28:2371-2376. 10.1023/a:1021009311977

Wang J, Hu P, Gao P, Tao J, and Luo Y. 2017a. Antennal transcriptome analysis and expression profiles of olfactory genes in Anoplophora chinensis. Scientific Reports 7. 10.1038/s41598-017-15425-2

Wang R, Li F, Zhang W, Zhang X, Qu C, Tetreau G, Sun L, Luo C, and Zhou J. 2017b. Identification and expression profile analysis of odorant binding protein and chemosensory protein genes in Bemisia tabaci MED by head transcriptome. Plos One 12:e0171739. 10.1371/journal.pone.0171739 PONE-D-16-49859 [pii]

Wang S-N, Peng Y, Lu Z-Y, Dhiloo KH, Gu S-H, Li R-J, Zhou J-J, Zhang Y-J, and Guo Y-Y. 2015. Identification and Expression Analysis of Putative Chemosensory Receptor Genes in Microplitis mediator by Antennal Transcriptome Screening. International Journal of Biological Sciences 11:737-751. 10.7150/ijbs.11786

Waris MI, Younas A, Ameen A, Rasool F, and Wang MQ. 2020. Expression Profiles and Biochemical Analysis of Chemosensory Protein 3 from Nilaparvata lugens (Hemiptera: Delphacidae). J Chem Ecol. 10.1007/s10886-020-01166-6 [pii]

Xu PX, Zwiebel LJ, and Smith DP. 2003. Identification of a distinct family of genes encoding atypical odorant-binding proteins in the malaria vector mosquito, Anopheles gambiae. Insect Molecular Biology 12:549-560. 10.1046/j.1365-2583.2003.00440.x

Zhang F, Merchant A, Zhao Z, Zhang Y, Zhang J, Zhang Q, Wang Q, Zhou X, and Li X. 2020. Characterization of MaltOBP1, a Minus-C Odorant-Binding Protein, From the Japanese Pine Sawyer Beetle, Monochamus alternatus Hope (Coleoptera: Cerambycidae). Frontiers in Physiology 11. 10.3389/fphys.2020.00212

Zhao Y, Wang F, Zhang X, Zhang S, Guo S, Zhu G, Liu Q, and Li M. 2016. Transcriptome and Expression Patterns of Chemosensory Genes in Antennae of the Parasitoid Wasp Chouioia cunea. Plos One 11:e0148159. 10.1371/journal.pone.0148159

Zheng R, Xia Y, and Keyhani NO. 2020. Sex-specific variation in the antennal proteome of the migratory locust. $J$ Proteomics 216:103681.

S1874-3919(20)30049-X

Peer] reviewing PDF | (2020:03:46684:1:1:NEW 18 Jun 2020) 
$610 \quad$ [pii]10.1016/j.jprot.2020.103681

611 Zheng X, Xie Z, Wang S, and Lin P. 2017. Determination of the protein expression profiles of 612 Propsilocerus akamusi (Tokunaga) Malpighian tubules response to cadmium stress by 613 iTRAQ coupled LC-MS/MS. Journal of Proteomics 164:85-93.

614

615

616 


\section{Table 1 (on next page)}

Table 1. Summary of sequencing reads after filtering. 
1 Table 1. Summary of sequencing reads after filtering.

\begin{tabular}{ccccccc}
\hline Sampl & $\begin{array}{c}\text { Total Raw } \\
\text { e }\end{array}$ & $\begin{array}{c}\text { Total Clean } \\
\text { Reads }(\mathrm{Mb})\end{array}$ & $\begin{array}{c}\text { Total Clean } \\
\text { Reads }(\mathrm{Mb})\end{array}$ & $\begin{array}{c}\text { Clean Reads } \\
\text { Bases }(\mathrm{Gb})\end{array}$ & $\begin{array}{c}\text { Q20 }(\%) \\
\text { Q30an Reads }(\%)\end{array}$ & $\begin{array}{c}\text { Clean Reads } \\
\text { Ratio(\%) }\end{array}$ \\
\hline A & 50.2 & 44.17 & 4.42 & 98.09 & 94.38 & 87.99 \\
B & 55.06 & 44.55 & 4.46 & 97.78 & 93.6 & 80.92 \\
C2 & 51.82 & 45.28 & 4.53 & 98.12 & 94.35 & 87.39 \\
\hline
\end{tabular}

2 
Table 2 (on next page)

Table 2. Quality metrics of transcripts 
2 Table 2. Quality metrics of transcripts

\begin{tabular}{llllllll}
\hline Sample & Total & Total & Mean & N50 & N70 & N90 & GC(\%) \\
& Number & Length & Length & & & & \\
\hline A & 28,143 & $24,421,248$ & 867 & 1,576 & 900 & 333 & 36.96 \\
B & 27,460 & $19,032,332$ & 693 & 1,231 & 637 & 263 & 38.99 \\
C2 & 25,567 & $22,046,525$ & 862 & 1,576 & 957 & 326 & 38.23 \\
\hline
\end{tabular}

3 N50: a weighted median statistic that $50 \%$ of the Total Length is contained in transcripts great 4 than or equal to this value. $\mathrm{GC}(\%)$ : the percentage of $\mathrm{G}$ and $\mathrm{C}$ bases in all transcripts. 
Table 3 (on next page)

Table 3. Quality metrics of Unigenes 
1 Table 3. Quality metrics of Unigenes

\begin{tabular}{llllllll}
\hline \multirow{2}{*}{ Sample } & Total & Total & Mean & N50 & N70 & N90 & GC(\%) \\
& Number & Length & Length & & & & \\
\hline A & 21,857 & $21,364,850$ & 977 & 1673 & 1015 & 389 & 37.12 \\
B & 19,975 & $16,078,029$ & 804 & 1375 & 765 & 313 & 38.62 \\
C2 & 19,355 & $19,187,584$ & 991 & 1667 & 1103 & 401 & 38.21 \\
All-Unigene & 27,609 & $28,312,956$ & 1025 & 1832 & 1146 & 396 & 38.23 \\
\hline
\end{tabular}

2 N50: a weighted median statistic that $50 \%$ of the TotalLength is contained in Unigenes great 3 than or equal to this value. $\mathrm{GC}(\%)$ : the percentage of $\mathrm{G}$ and $\mathrm{C}$ bases in all Unigenes.

4 
Table 4 (on next page)

Table 4. Summary of functional annotation result. 
1 Table 4. Summary of functional annotation result.

\begin{tabular}{ccc}
\hline Values & Number & Percentage \\
\hline Total & 27609 & $100.00 \%$ \\
NR & 17311 & $62.70 \%$ \\
NT & 7936 & $28.74 \%$ \\
Swissprot & 14614 & $52.93 \%$ \\
KEGG & 14341 & $51.94 \%$ \\
COG & 7986 & $28.93 \%$ \\
Interpro & 14752 & $53.43 \%$ \\
GO & 7501 & $27.17 \%$ \\
Overall & 18544 & $67.17 \%$ \\
\hline
\end{tabular}

2 Overall: the number of Unigenes which be annotated with at least one functional database. 
Table 5 (on next page)

Table 5. Quality metrics of predicted CDS 
1 Table 5. Quality metrics of predicted CDS

\begin{tabular}{llllllll}
\hline Software & $\begin{array}{l}\text { Total } \\
\text { Number }\end{array}$ & $\begin{array}{l}\text { Total } \\
\text { Length }\end{array}$ & $\begin{array}{l}\text { Mean } \\
\text { Length }\end{array}$ & N50 & N70 & N90 & GC(\%) \\
\hline Blast & 17,522 & $15,693,411$ & 895 & 1,350 & 912 & 417 & 41.83 \\
ESTScan & 2,857 & 974,223 & 340 & 339 & 261 & 213 & 44.69 \\
Overall & 20,379 & $16,667,634$ & 817 & 1,290 & 834 & 348 & 42.00 \\
\hline
\end{tabular}

2 N50: a weighted median statistic that $50 \%$ of the TotalLength is contained in CDS great than or 3 equal to this value. GC (\%): the percentage of $\mathrm{G}$ and $\mathrm{C}$ bases in all CDS. 
Figure 1

Fig. 1 The movement situation (mean $\pm \mathrm{SD}$ ) of $P$. akamusi.

(A) The movement situation (mean $\pm \mathrm{SD}$ ) of male $P$. akamusi when attracted to female. (B) The movement situation (mean $\pm \mathrm{SD}$ ) of female $P$. akamusi when attracted to male.

A

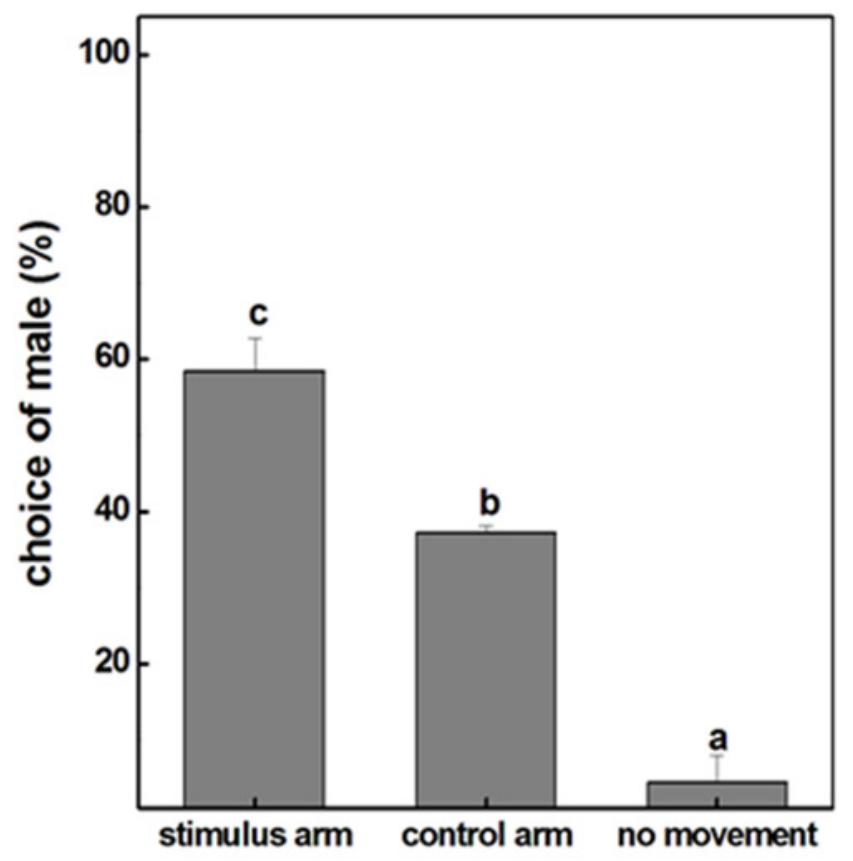

B

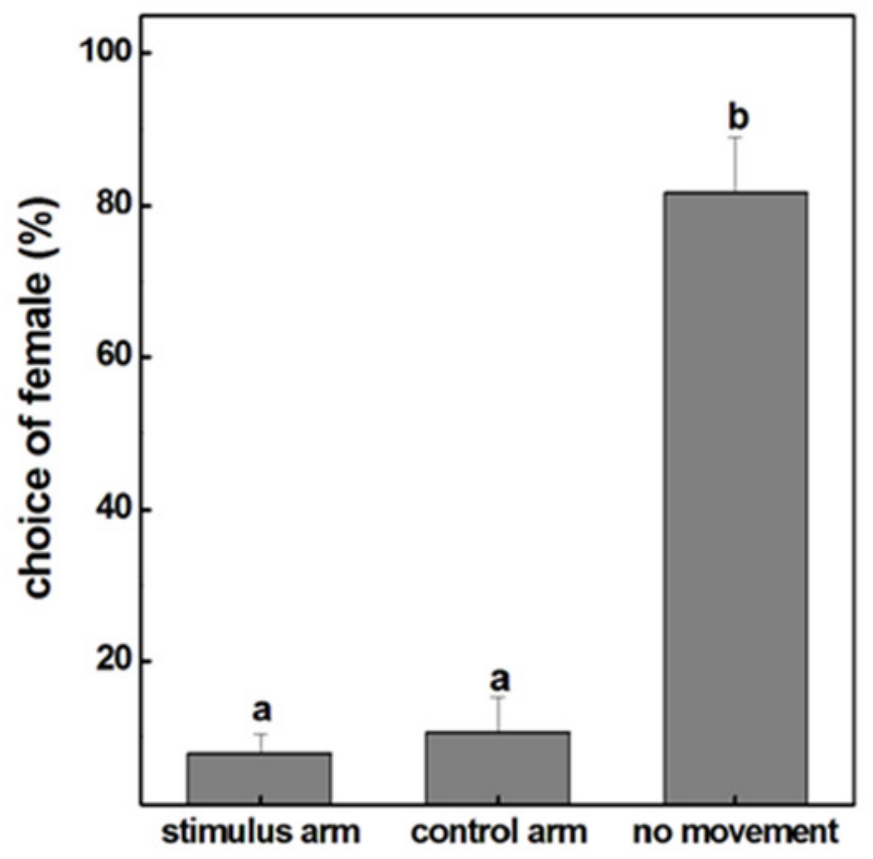


Figure 2

Fig. 2 The homology searching against NR database.

\section{Species Distribution}

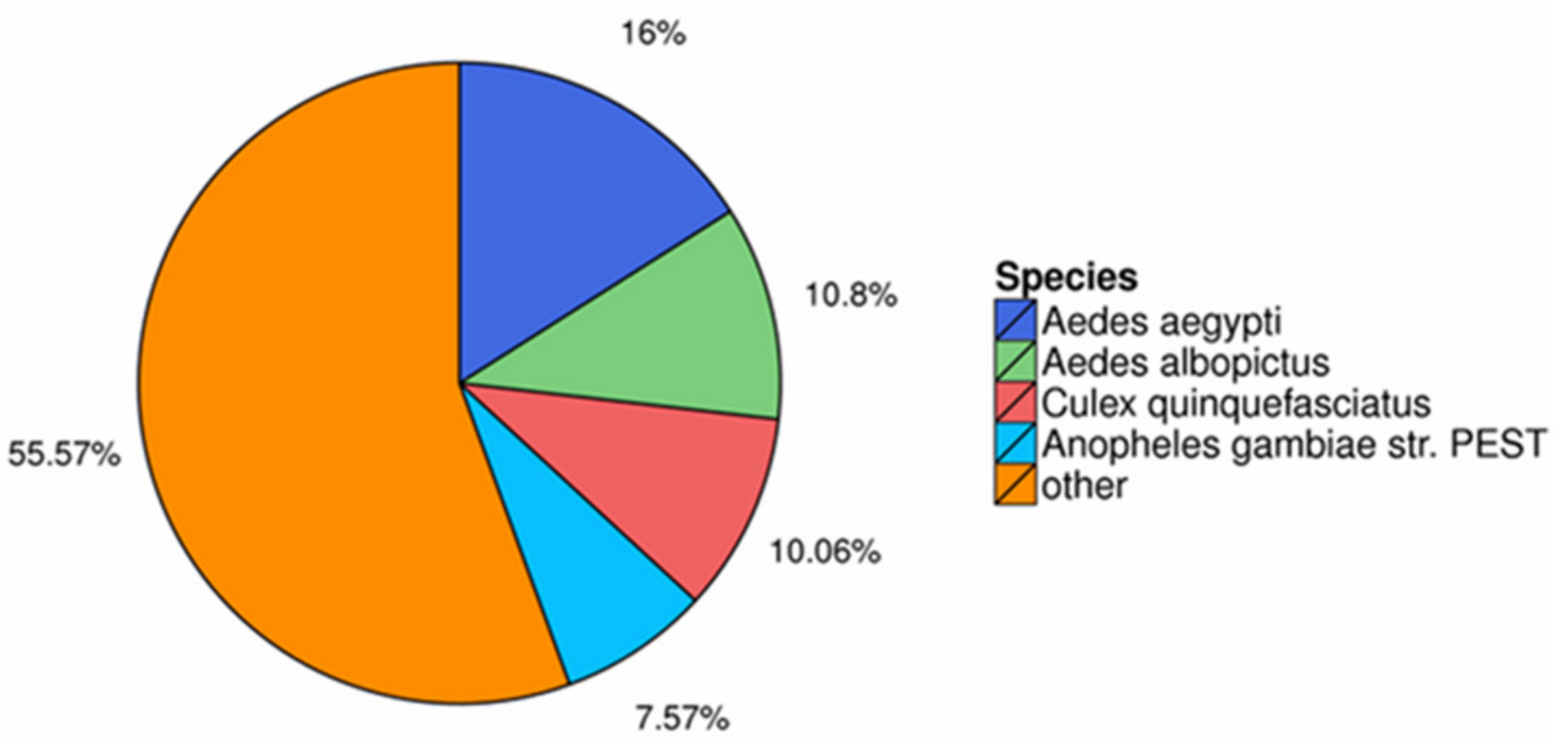




\section{Figure 3}

Fig. 3 Functional annotation for Unigenes of $P$. akamusi.

(A) Functional distribution of $G O$ annotation. $X$ axis represents the number of Unigenes. $Y$ axis represents the Gene Ontology functional category. (B) Functional distribution of KEGG annotation. $X$ axis represents the number of Unigenes. $Y$ axis represents the KEGG functional category. (C) Functional distribution of $\mathrm{COG}$ annotation. $X$ axis represents the number of Unigenes. $Y$ axis represents the COG functional category. (D)Venn diagram between NR, COG, KEGG, Swissprot and Interpro. 
A

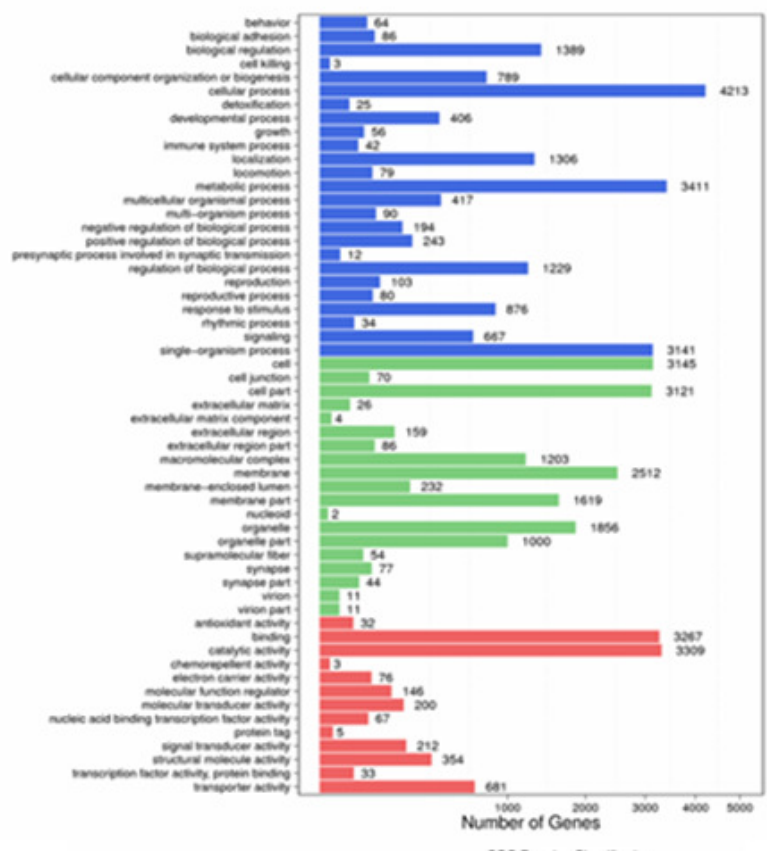

C

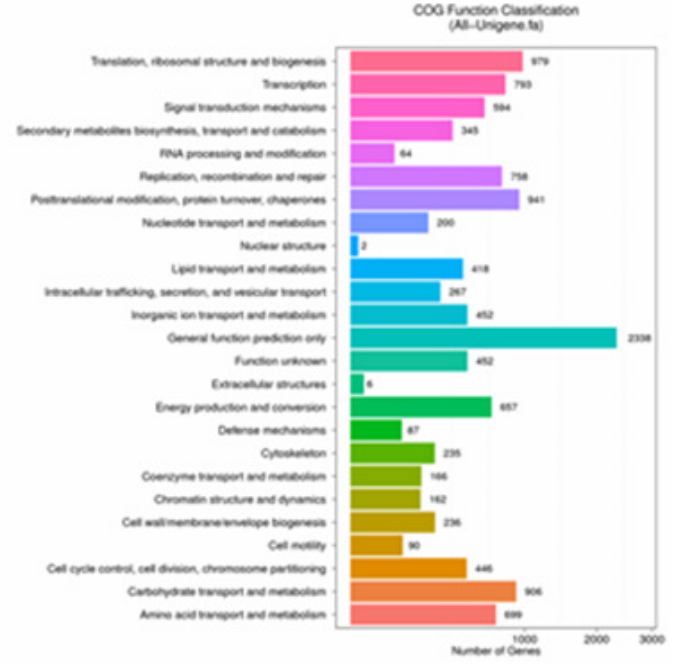

B

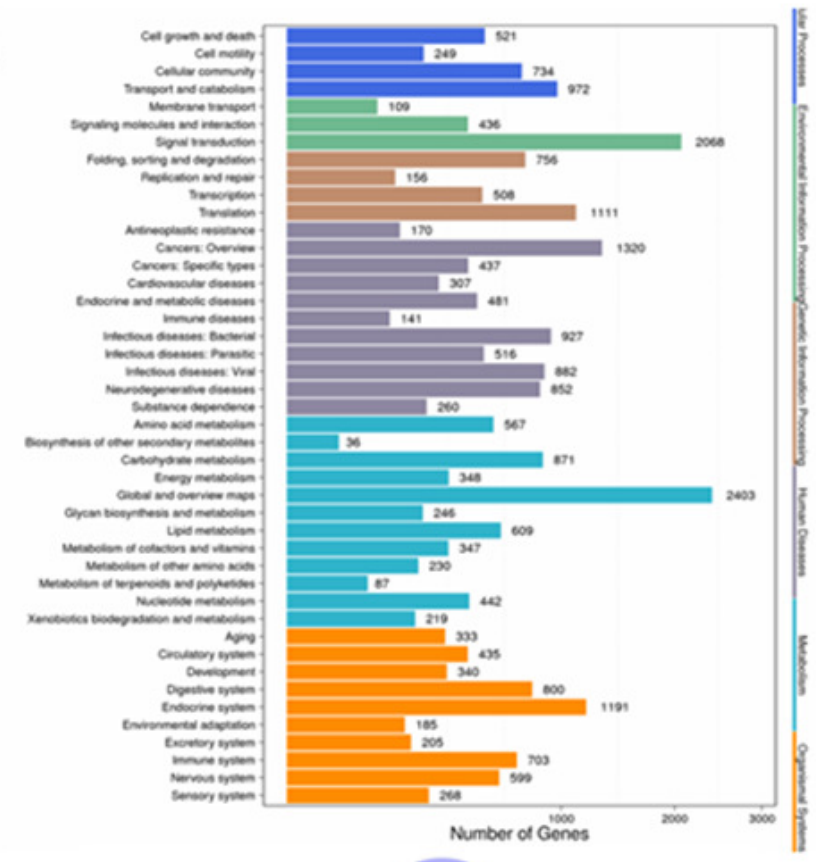

D

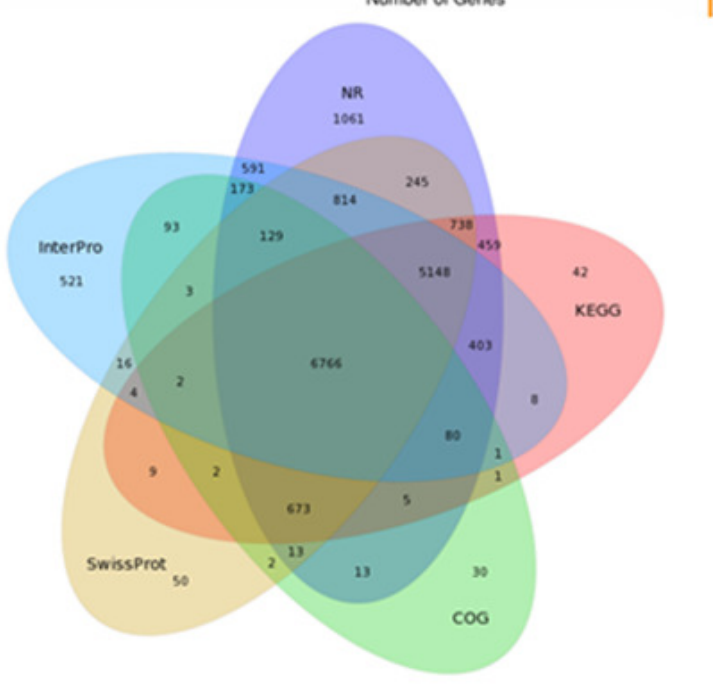


Figure 4

Fig. 4 CDS length distribution of $P$. akamusi.

$X$ axis represents the length of $C D S$. $Y$ axis represents the number of $C D S$.

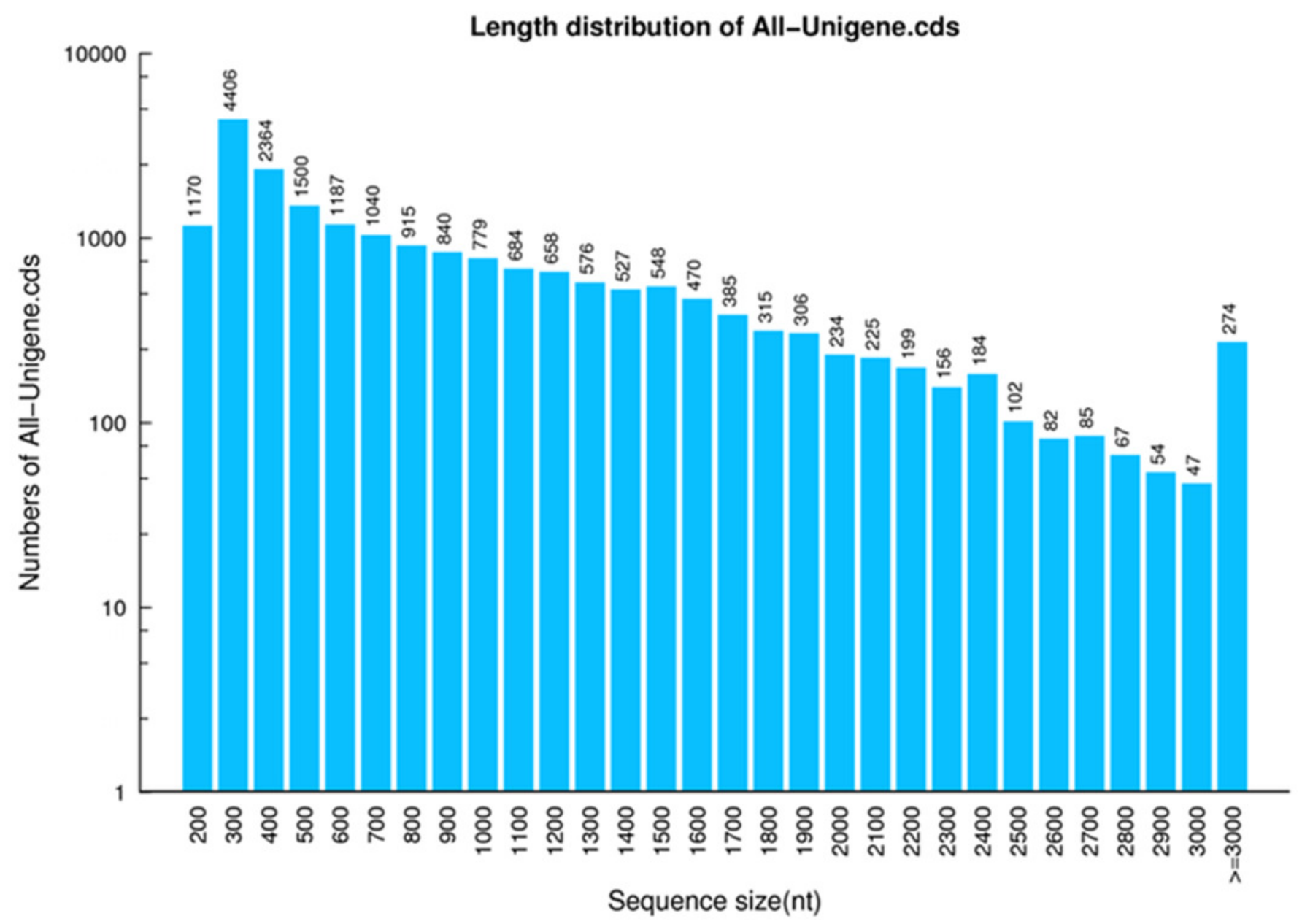




\section{Figure 5}

Fig. 5 Differentially expressed genes (DEGs) in P. akamusi. (A) Summary of DEGs.

$X$ axis represents comparing samples. $Y$ axis represents the number of DEGs. Red color represents up-regulated DEGs while blue color represents down-regulated ones. Group: A, Heads; B, Leg; C, Antennae. (B) Venn diagram showing intersections and disjunctions of genes expressed in heads, legs and antennae.

A

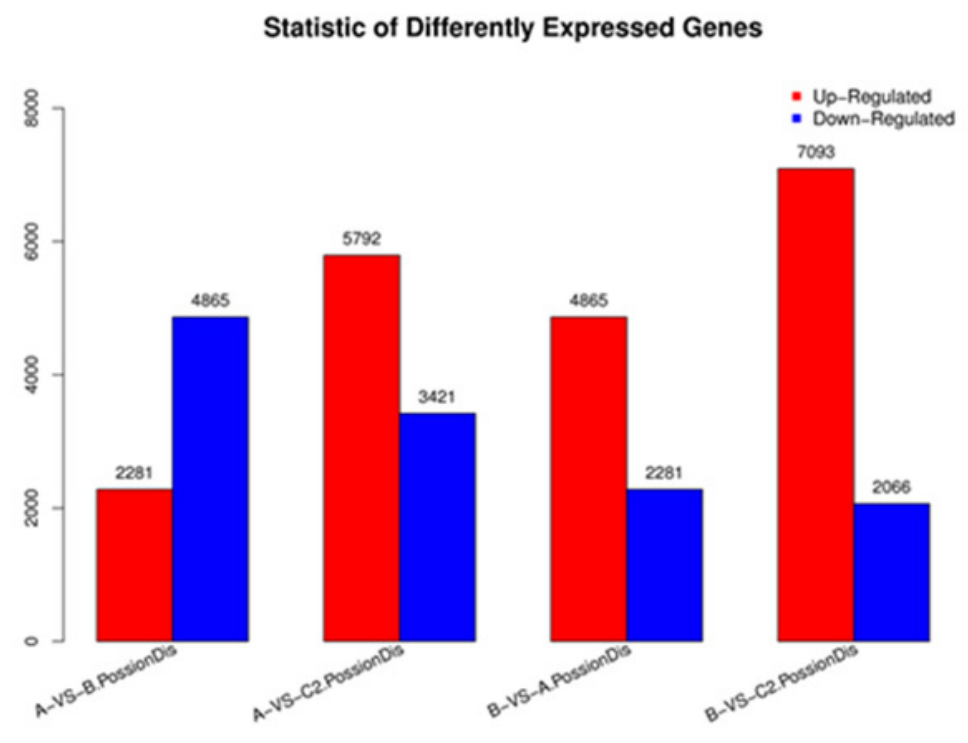

B

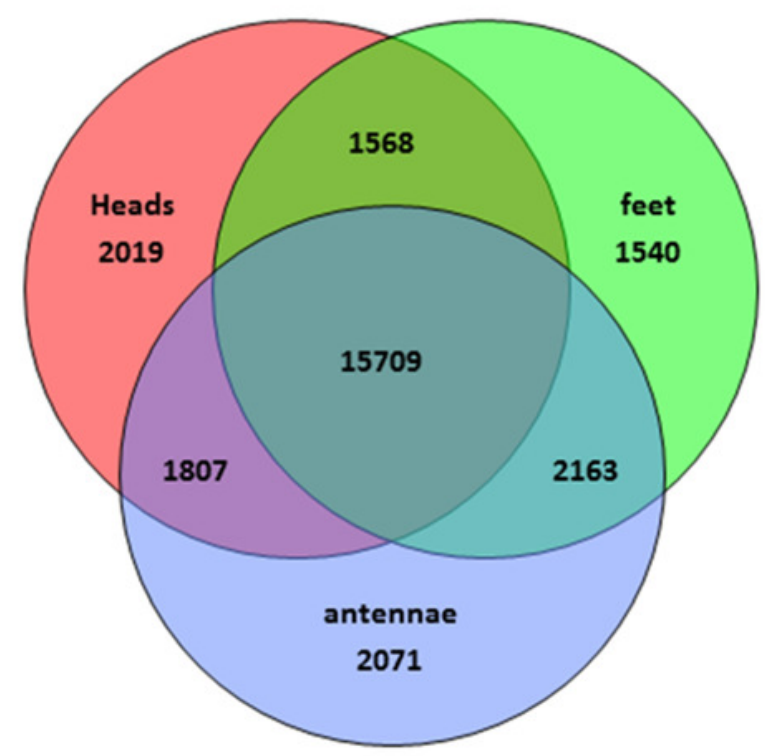


Figure 6

Fig. 6 P. akamusi OBPs transcript levels in different tissues measured by RT-qPCR.

"AN" for antennae; " HE " for head; " LE " for leg. The tubulin was used to normalize transcript levels in each sample. The standard error is represented by the error bar, and the different letters $(a, b, c, d)$ above each bar denote significant differences $(p<0.05)$.

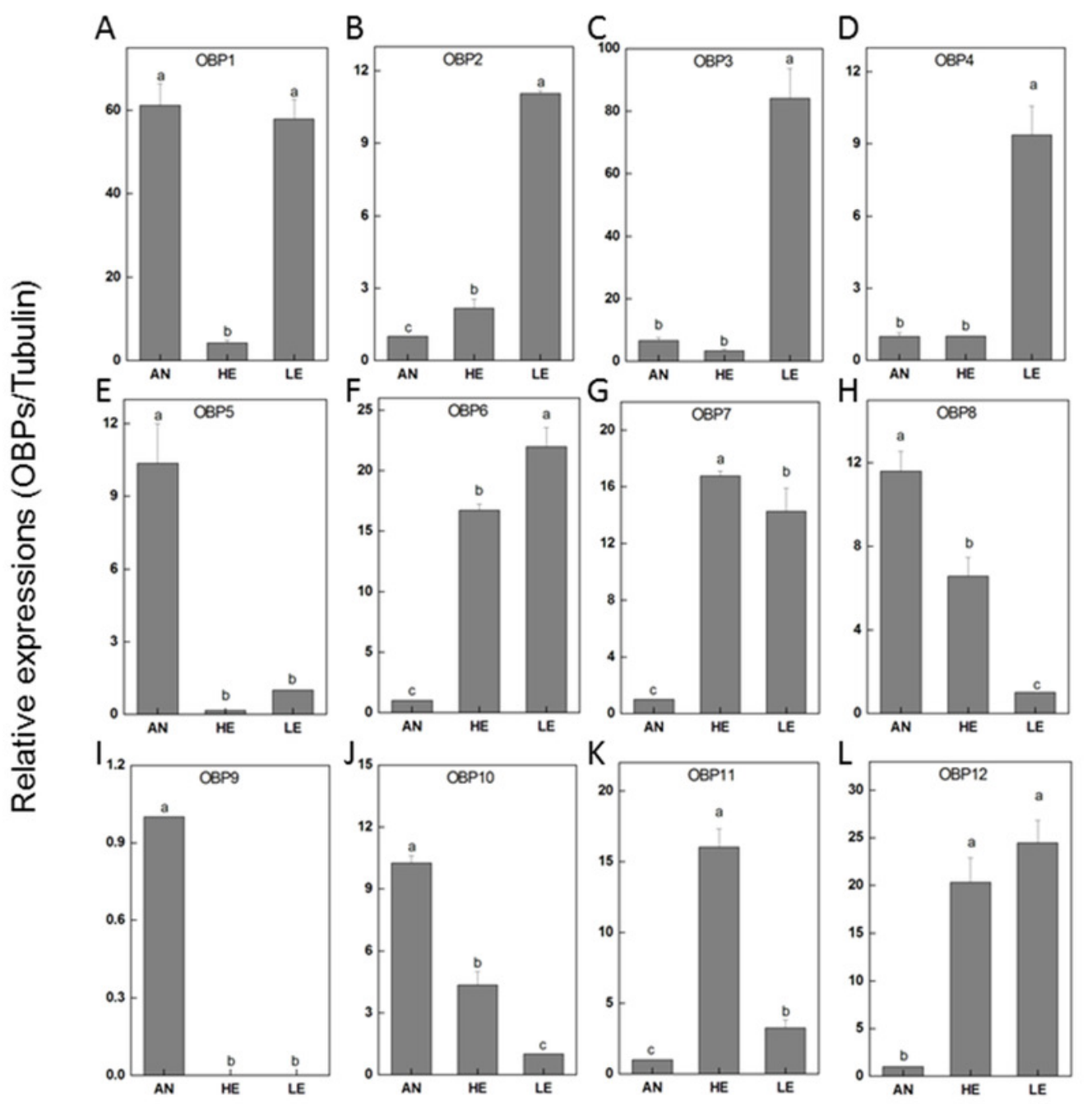


Figure 7

Fig. 7 P. akamusi ORs transcript levels in different tissues measured by RT-qPCR.

"AN" for antennae; " HE " for head; " LE " for leg. The tubulin was used to normalize transcript levels in each sample. The standard error is represented by the error bar, and the different letters $(a, b, c, d)$ above each bar denote significant differences $(p<0.05)$.

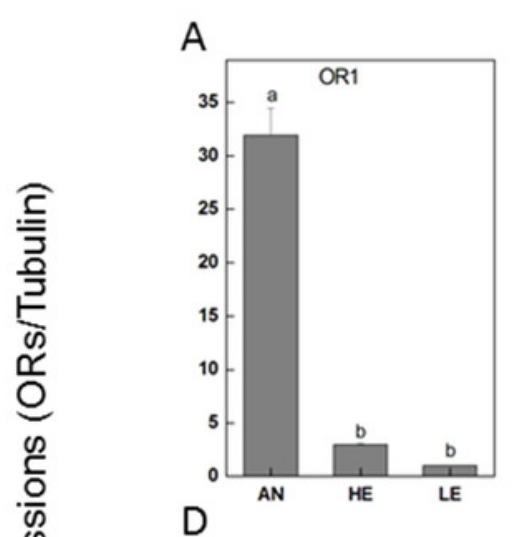

B
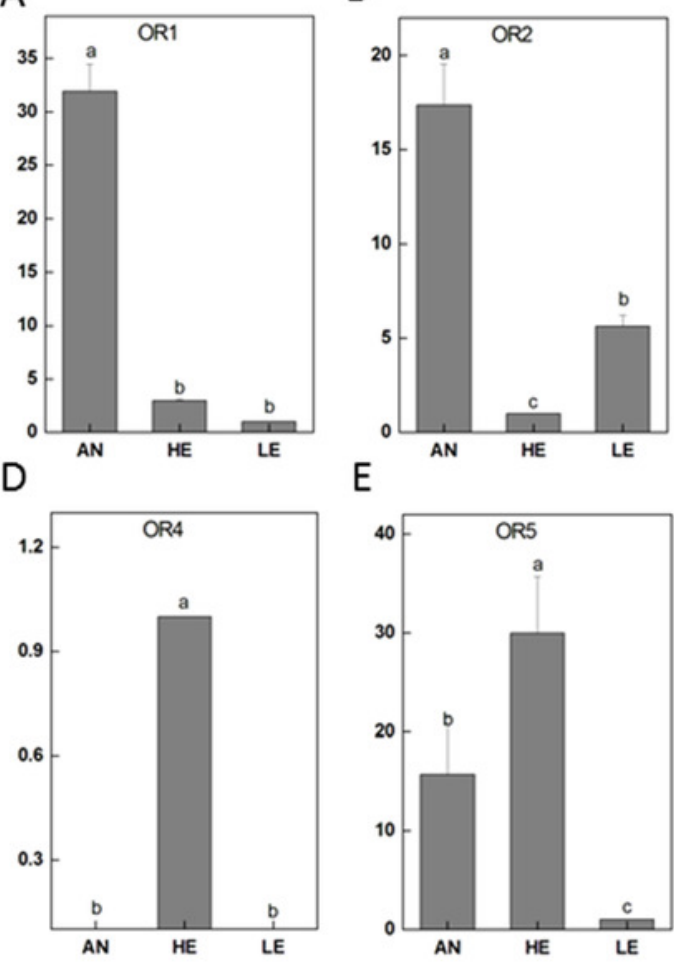

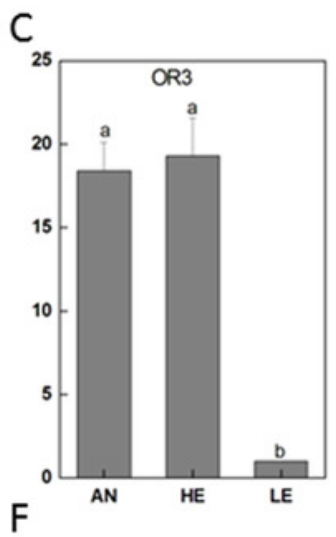

$\mathrm{F}$
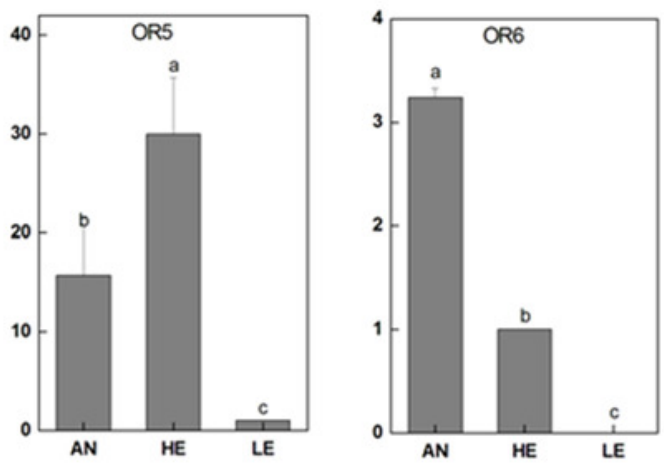
Figure 8

Fig. 8 P. akamusi GRs transcript levels in different tissues measured by RT-qPCR.

"AN" for antennae; " HE " for head; " LE " for leg. The tubulin was used to normalize transcript levels in each sample. The standard error is represented by the error bar, and the different letters $(a, b, c, d)$ above each bar denote significant differences $(p<0.05)$.
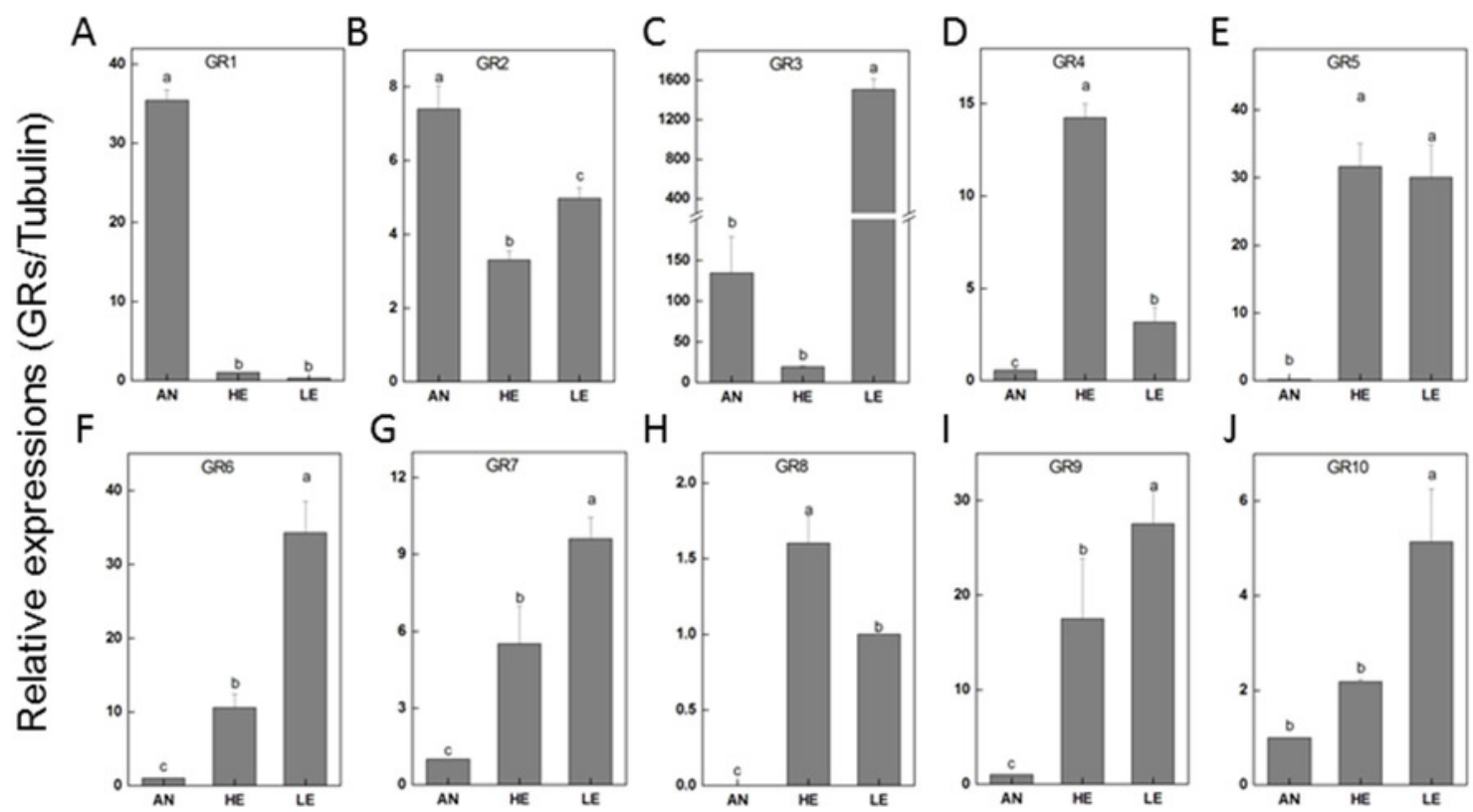
Figure 9

Fig. 9 P. akamusi IRs transcript levels in different tissues measured by RT-qPCR.

"AN" for antennae; " HE " for head; " LE " for leg. The tubulin was used to normalize transcript levels in each sample. The standard error is represented by the error bar, and the different letters $(a, b, c, d)$ above each bar denote significant differences $(p<0.05)$.
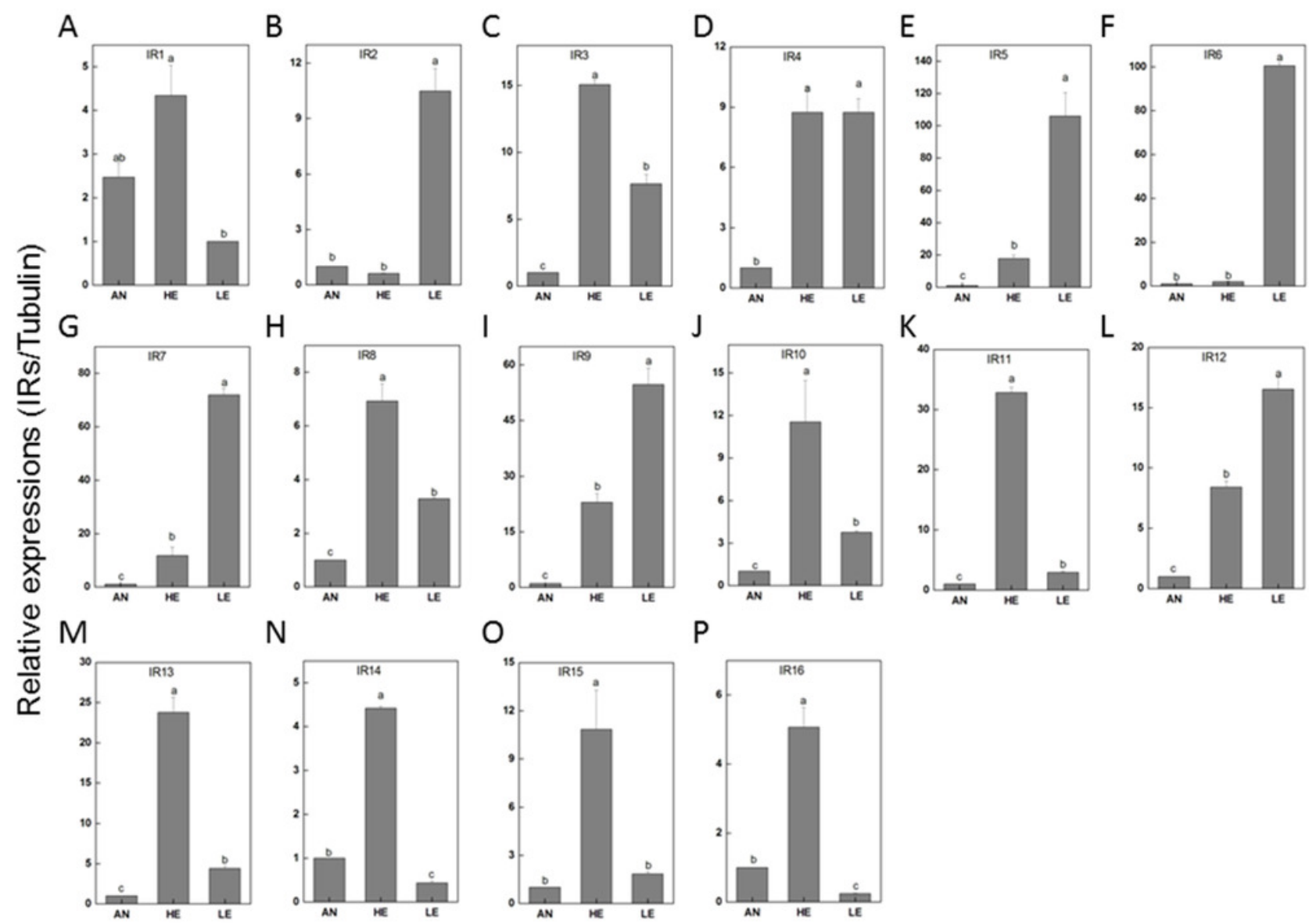
Figure 10

Fig. 10 P. akamusi CSP1 and SNMP1 transcript levels in different tissues measured by RT-qPCR.

"AN" for antennae; "HE " for head; "LE " for leg. The tubulin was used to normalize transcript levels in each sample. The standard error is represented by the error bar, and the different letters $(a, b, c, d)$ above each bar denote significant differences $(p<0.05)$.

A

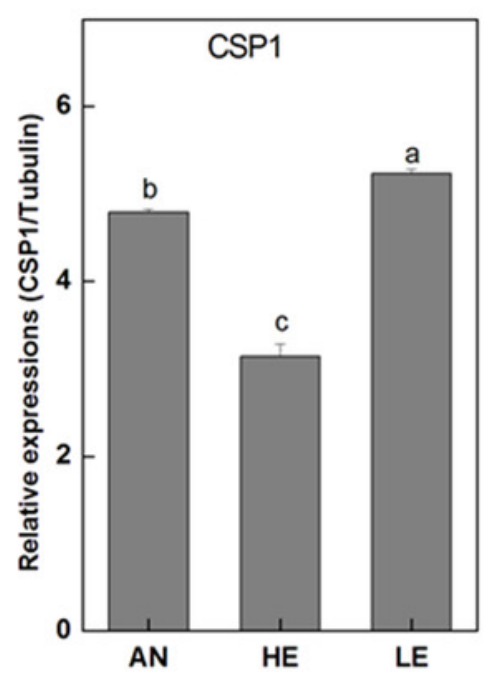

B

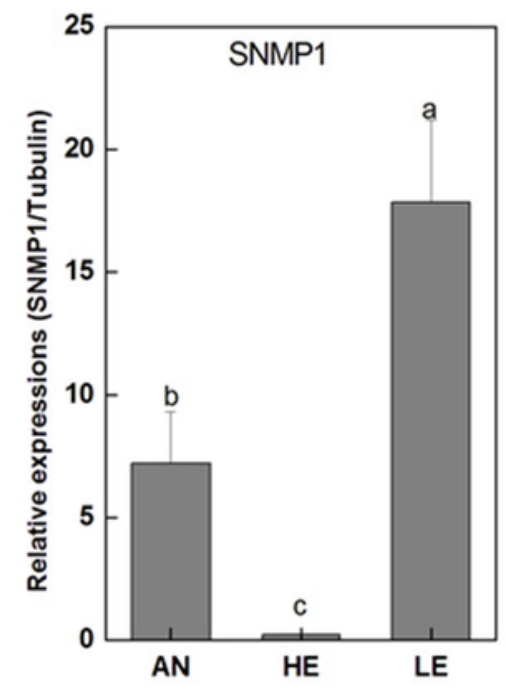

